\title{
EDITORIAL
}

\section{Metropolen und Metropolregionen}

\author{
Andreas Klee
}

Online publiziert: 28. Januar 2014

(C) Springer-Verlag Berlin Heidelberg 2014

Metropolregion - welch schillernder Begriff! Er hat wie nur ganz wenig andere Begriffe den raumwissenschaftlichen Diskurs in den letzten Jahren geprägt. Keine großstädtisch geprägte Region in Deutschland kommt heute ohne dieses Attribut aus. Blickt man aufmerksam in die Fachzeitschriften, auf die Websites der deutschen Großstädte, in die Tagesberichterstattung der Zeitungen, ist man geneigt, von einem inflationären Gebrauch des Begriffs zu sprechen.

Es waren wohl die neuen „Leitbilder und Handlungsstrategien für die Raumentwicklung in Deutschland“, von der Ministerkonferenz für Raumordnung 2006 beschlossen, die die wissenschaftliche, politische, aber auch alltagsweltliche Diskussion über Metropolregionen entscheidend befördert hat. Prominent in einem Leitbild verankert, sollten Metropolregionen stärker in den raumplanerischen und raumentwicklungspolitischen Fokus gerückt werden. Ihre Bedeutung bei der Generierung von wirtschaftlichem Wachstum und als Zentren hochrangiger kultureller, politischer und administrativer Funktionen sollte herausgestellt werden. Parallel zur Diskussion der Metropolregionen im Rahmen der neuen Leitbilder sind bis Mitte der 2000er Jahre in Deutschland elf „Europäische Metropolregionen“ entstanden. Sie unterscheiden sich erheblich - hinsichtlich ihres räumlichen Umgriffs, ihres großstädtischen Charakters, ihrer rechtlichen und administrativen Verfasstheit, ihrer Aufgaben und, nicht zuletzt, ihrer Sichtbarkeit in den Medien und bei der Bevölkerung sowie der Identifizierung der jeweiligen Bevölkerung mit „,ihrer“ Metropolregion.

Dr. A. Klee $(\bowtie)$

Akademie für Raumforschung und Landesplanung,

Leibniz-Forum für Raumwissenschaften,

Hohenzollernstraße 11, 30161 Hannover, Deutschland

E-Mail: klee@arl-net.de
Vieles war in den letzten Jahren zu beobachten: Die Entwicklung von Metropolregionen, die es vermochten, ein metropolregionales Bewusstsein bei vielen Akteuren zu schaffen, neue Projekte anzustoßen, die Region im weltweiten Standortwettbewerb besser zu positionieren und ein Denken in regionalen Zusammenhängen voranzubringen. Es war aber auch zu beobachten, dass Metropolregionen möglicherweise aufgrund politischen Drucks - entstanden sind, bei denen man fragen möchte, zu welchem Zweck sie gebildet wurden. In nicht wenigen Fällen sind die Metropolregionen räumlich sehr großzügig geschnitten, umfassen ganze Bundesländer, einschließlich großer ländlicher Räume, oder bestehen aus Teilräumen, die bis heute nicht zueinandergefunden haben, vielleicht auch gar nicht zusammenfinden wollen. Hier verkommt der Begriff der Metropolregion zum bloßen Etikett ohne großen Mehrwert.

Man kann festhalten, dass in den letzten zehn Jahren viel über Metropolregionen geschrieben wurde, in dieser wie in anderen raumwissenschaftlichen Zeitschriften, in Tages- und Wochenzeitungen. Zumeist ging es darum, die Bedeutung der Metropolregionen für die Raumentwicklung herauszustellen, die jeweilige Governance zu analysieren, ihre Wirkungen im Rahmen einer Standortpolitik zu erfassen. Eine Frage, die in all diesen Diskussionen zumeist ausgeblendet blieb, ist die nach der empirischen Evidenz von Metropolregionen und ihrer Funktionen. Anders ausgedrückt: Wie verhält es sich mit den „metropolitanen Funktionen" in den Metropolregionen? Welche sind in welcher Intensität vorhanden und in welchem Zusammenhang stehen sie zum Attribut der Metropolregionen?

Hierzu gibt es in Deutschland - ganz im Gegensatz zur internationalen Diskussion - nur wenige konzeptionelle Überlegungen und empirische Arbeiten. Eine der wenigen Autorinnen ist Kati Volgmann, die in dieser Ausgabe von „Raumforschung und Raumordnung“ über die Entwicklung 
metropolitaner Funktionen im deutschen Städtesystem schreibt. Sie prüft, welche Städte metropolitane Funktionen haben und wie sich diese im Hinblick auf die Prozesse der räumlichen Konzentration und funktionalen Spezialisierung zwischen 1995 und 2010 entwickelt haben. Sie arbeitet auf Basis einer umfangreichen Datenanalyse heraus, dass aufgrund von Agglomerationsvorteilen, beispielsweise die räumliche Nähe oder Entwicklungsvorsprünge, die ,großen“" Metropolräume - der Begriff Metropolregion wird bewusst nicht verwendet - als Gewinner der Metropolisierungsprozesse hervorgehen. Neben diesen Konzentrationsprozessen zugunsten der ,großen“ Metropolräume stellt Kati Volgmann auch eine zunehmende Spezialisierung der Metropolräume fest. Sie interpretiert Spezialisierungsprozesse als funktionsspezifische Lokalisationseffekte, die mit Kostenvorteilen durch die Verringerung von Transport- und Transaktionskosten sowie die Bedeutung räumlicher Nähe zum Wissensaustausch und zur Wissensabsorption begründet werden können. Insgesamt gesehen trägt sie mit dieser fundierten empirischen Analyse dazu bei, dass der Diskurs über Metropolregionen ein Stück weit versachlicht wird und damit der Metropolregionsbegriff mit Inhalten gefüllt werden kann.

Das vorliegende Heft von „Raumforschung und Raumordnung" bietet eine Reihe weiterer interessanter Aufsätze. Ingo Mose, Marta Jacuniak-Suda und Georg Fiedler arbeiten in ihrem Beitrag „Regional Governance-Stile in Europa. Eine vergleichende Analyse von Steuerungsstilen ausgewählter LEADER-Netzwerke in Extremadura (Spanien), Warmińsko-Mazurskie (Polen) und Western Isles (Schottland)" unterschiedliche Auffassungen und Konzeptionierungen von Regional Governance auf europäischer Maßstabsebene heraus. Dies dient dazu, von der großen Zahl verschiedener kontextueller Interpretationen von Governance zu abstrahieren und damit eine weiterführende Perspektive für Analyse und Vergleich von Regional-Governance-Phänomenen $\mathrm{zu}$ entwickeln. Sie thematisieren, wie andere Autoren auch, dass Governance-Strukturen und deren zunehmender Bedeutungsgewinn vielfach als ein paradigmatischer Wandel für die Regionalentwicklung gesehen werden können. Allerdings zeigen ihre empirischen Arbeiten in drei europäischen Regionen, dass sich die Governance-Strukturen unterscheiden und dass sich spezi- fische „Stile“ identifizieren lassen und eben keine ähnlichen Muster.

Dörthe Brinker und Heidi Sinning untersuchen in ihrem Beitrag „Wohnraumversorgung und Wohnqualität einkommensschwacher Haushalte" das Quartier Sonnenberg in der sächsischen Großstadt Chemnitz. Sie fokussieren dabei auf zwei Strategieansätze: der Kooperation zwischen Wohnungswirtschaft und Stadtentwicklung sowie der integrierten Quartiersentwicklung. Am Beispiel des Quartiers loten sie entsprechende Handlungsmöglichkeiten und Grenzen der Wohnraumversorgung exemplarisch aus und ordnen sie in den wissenschaftlichen Kontext einer kooperativen und sozial ausgleichenden Stadtentwicklungspolitik ein. Unter anderem fordern sie, kommunale Konzepte zur Wohnraumversorgung von Haushalten mit niedrigen Einkommen zu erstellen, die Kooperationsbeziehungen zwischen der Wohnungswirtschaft und den Städten zu intensivieren, hinsichtlich der Sozialstruktur und Einkommensverhältnisse gemischte Quartiere zu sichern und dort eine integrierte Quartierspolitik zu unterstützen.

Schließlich befassen sich Christian Diller und Keno Frank mit räumlichen Aspekten der Schulversorgung aus Nutzersicht und präsentieren Ergebnisse einer Eltern-Schüler-Befragung in den Kreisen Steinburg und Dithmarschen in Schleswig-Holstein. Sie fragen danach, welche Rolle angesichts gestiegenen Bildungswettbewerbs, Leistungsdrucks in den Schulen und einem immer weiter qualitativ differenzierten Bildungsangebot räumliche Aspekte im Bildungsverhalten von Schülern und deren Eltern überhaupt noch spielen, und ob die räumliche Nähe von Schulen angesichts allgemein vergrößerter Aktionsräume überhaupt noch wichtig ist. Hierzu wurden mit qualitativen und quantitativen Methoden eine Vielzahl von Schülern, Lehrern und Eltern befragt.

Zuletzt eine Nachricht in eigener Sache: Nach zwei Jahren Tätigkeit als Schriftleiterin dieser Zeitschrift ist Hendrikje Wehnert vom Leibniz-Institut für ökologische Raumentwicklung (IÖR) in Dresden zum Jahreswechsel 2013/2104 turnusmäßig ausgeschieden. Für die geleistete Arbeit danken wir ihr sehr herzlich. Ihre Position als Schriftleiter für die Jahre 2014 und 2015 nimmt Wladimir Sgibnev vom Leibniz-Institut für Länderkunde (IfL) in Leipzig ein. Herzlich willkommen - wir freuen uns auf die Zusammenarbeit! 